\title{
GCU
}

Glasgow Caledonian

University

University for the Common Good

\section{An in vitro evaluation of the efficacy of tedizolid: implications for the treatment of skin and soft tissue infections}

Delpech, Pierre; Aleryan, Muna; Jones, Brian; Gemmell, Curtis ; Lang, Susan

Published in:

Diagnostic Microbiology and Infectious Disease

DOI:

10.1016/j.diagmicrobio.2018.01.006

Publication date:

2018

Document Version

Author accepted manuscript

Link to publication in ResearchOnline

Citation for published version (Harvard):

Delpech, P, Aleryan, M, Jones, B, Gemmell, C \& Lang, S 2018, 'An in vitro evaluation of the efficacy of tedizolid: implications for the treatment of skin and soft tissue infections', Diagnostic Microbiology and Infectious Disease, vol. 91, no. 1, pp. 93-97. https://doi.org/10.1016/j.diagmicrobio.2018.01.006

\section{General rights}

Copyright and moral rights for the publications made accessible in the public portal are retained by the authors and/or other copyright owners and it is a condition of accessing publications that users recognise and abide by the legal requirements associated with these rights.

Take down policy

If you believe that this document breaches copyright please view our takedown policy at https://edshare.gcu.ac.uk/id/eprint/5179 for details of how to contact us. 
$7 \quad{ }^{\mathrm{a} D e p a r t m e n t}$ of Life Sciences, School of Health and Life Sciences, Glasgow Caledonian

8 University, Glasgow, G4 OBA, UK.

$9 \quad{ }^{b}$ Consultant Medical Microbiologist and Head of Microbiology, NHS Greater Glasgow and

10 Clyde, Glasgow Royal Infirmary, 84 Castle Street, Glasgow, G4 0SF, UK.

$11{ }^{\mathrm{c}}$ Division of Infection, Immunity and Inflammation, University of Glasgow, University

12 Avenue, Glasgow, G12 8QQ.

13

14

15

16

17 Department of Life Sciences, School of Health and Life Sciences,

18 Glasgow Caledonian University,

19 Cowcaddens Road,

20 Glasgow, G4 OBA.

$21 \quad$ Tel: +44 1413318092.

22

\section{* Corresponding Author}

Sue Lang,

Email: sue.lang@gcu.ac.uk. 
Abstract

Skin and soft tissue infections (SSTI) are among the most commonly occurring infections and evidence suggests that these are increasing world-wide. The aetiology is diverse, but Staphylococcus aureus predominate and these are often resistant to antimicrobials that were previously effective. Tedizolid is a new oxazolidinone-class antibacterial indicated for the treatment of adults with SSTI caused by Gram-positive pathogens, including S. aureus.

The aim of this study was to evaluate the in vitro efficacy of tedizolid in comparison to other clinically used antibacterials against antibiotic sensitive- and resistant-staphylococci, grown in planktonic cultures and as biofilms reflecting the growth of the microorganism during episodes of SSTI.

Against a panel of 66 clinical staphylococci, sensitivity testing revealed that a lower concentration of tedizolid was required to inhibit the growth of staphylococci compared to linezolid, vancomycin and daptomycin; with the tedizolid $\mathrm{MIC}_{50}$ being 8-fold (S. aureus) or 4fold (S. epidermidis) below that obtained for linezolid. In addition, cfr+ linezolid-resistant strains remained fully susceptible to tedizolid. Against S. aureus biofilms, 10×MIC tedizolid was superior or comparable with 10×MIC comparator agents in activity, and superior to 10×MIC linezolid against those formed by S. epidermidis (65 vs. 33\% reduction, respectively).

Under flow-conditions both oxazolidinones at 10xMIC statistically out-performed vancomycin in their ability to reduce the viable cell count within a $S$. aureus biofilm with fewer the $12 \%$ of cells surviving compared to $63 \%$ of cells. 
In conclusion, tedizolid offers a realistic lower-dose alternative agent to treat staphylococcal

47 SSTI, including infections caused by multi-drug resistant strains.

49 Keywords: skin and soft tissue infections, tedizolid, linezolid, staphylococcus, biofilm,

50 minimum inhibitory concentration

51

52 Abbreviations:

53 CFU, colony forming unit

54 DAP, daptomycin

55 DMSO, dimethyl sulfoxide

56 EUCAST, European Committee on Antimicrobial Susceptibility Testing

57 GMO, genetically modified organism

$58 \quad$ LZD, linezolid

59 MRSA, methicillin resistant Staphylococcus aureus

60 MSSA, methicillin sensitive Staphylococcus aureus

61 MIC, minimum inhibitory concentration

62 MHB, Mueller-Hinton broth

63 PBS, phosphate buffered saline

64 SSTI, skin and soft tissue infection

65 TZD, tedizolid

66 VAN, vancomycin

67 VISA, vancomycin intermediate susceptibility S. aureus 


\section{Introduction}

Skin and soft tissue infections (SSTIs) are common within both hospitalised patients and individuals within the community, yet providing a suitable treatment remains a clinical challenge. Published national and international guidelines for the treatment of SSTIs broadly agree [1]. The United Kingdom's National Institute for Clinical Excellence (NICE) guidelines, for example, emphasise the importance of using empirical treatment effective against methicillin resistant Staphylococcus aureus (MRSA) [1]. With the subsequent knowledge of bacterial cultures, treatment can be de-escalated to a narrow spectrum agent, preferably with oral administration allowing treatment to continue in the community. In reality, a microbiological diagnosis may not be available and initial therapy inadequate leading to clinical failure, recurrence of infection and readmission to hospital increasing the overall length of patient stay. Complicating therapy further, resistant Staphylococcus aureus can be responsible for in the region of half of complicated SSTIs, yet empirical therapy is often not appropriate for these microorganisms [2].

Currently vancomycin, linezolid, daptomycin, ceftaroline and telavancin are among those antibacterials recommended for the treatment of severe SSTIs with other agents in reserve for milder infections [3]. Newer agents are becoming available, including tedizolid, dalbavancin and oritavancin, but clinical evidence for the role of these agents is limited and needs to be provided if future guidelines are to be established [4]. Tedizolid phosphate (Sivextro ${ }^{\circledR}$ ) is a next-generation oxazolidinone antibacterial approved for the treatment of adults with acute SSTIs caused by susceptible Gram-positive microorganisms, including staphylococci [5]. The spectrum of activity is similar to linezolid, 
92 though activity is retained against some strains that are resistant to linezolid [6]. Similar in

93 mode of action to other oxazolidinones, antibacterial activity is mediated by inhibiting

94 protein synthesis [7].

95 Tedizolid is a new drug approved for the treatment of SSTIs in a number of countries,

96 including the United States, Cananda and the European Union [4]. The aim of this study was

97 to evaluate the in vitro efficacy of tedizolid in comparison to other clinically used

98 antibacterials against antibiotic sensitive and resistant staphylococci, grown in planktonic

99 cultures and as biofilms reflecting the growth of the microorganism during episodes of SSTI. 


\section{Material and Methods}

\subsection{Strains, culture conditions and preparation of antibiotics}

103 The study included 66 clinical staphylococcal isolates: 27 methicillin sensitive S. aureus (MSSA) (including two linezolid-resistant), 27 MRSA (including two linezolid-resistant) and 12 Staphylococcus epidermidis (including two linezolid-resistant). Except the six linezolidresistant strains (provided by J. Mingorance, Madrid), all strains were supplied by the Scottish MRSA Reference Laboratory, Glasgow (Supplementary Table 1).

All experiments were performed in Mueller-Hinton broth (MHB, Oxoid); for testing with daptomycin the medium was supplemented with $50 \mathrm{mg} / \mathrm{L} \mathrm{Ca}^{2+}[8]$.

Tedizolid and linezolid were gifted by MSD and Pfizer, respectively (MSD, Hertfordshire, UK; Pfizer Ltd, Surrey, UK). Vancomycin and daptomycin were purchased from Sigma-Aldrich (Dorset, UK). Stock solutions of tedizolid were prepared in dimethyl sulfoxide (DMSO, 1,600 $\mathrm{mg} / \mathrm{L}$ ) prior to 2-fold dilutions in DMSO as per the supplier's guidelines. Other antibiotic stocks of $10,000 \mathrm{mg} / \mathrm{L}$ (except linezolid 1,000 mg/L) were prepared using distilled water and used or stored at $-20^{\circ} \mathrm{C}$ for a maximum of two weeks.

\subsection{Antibiotic susceptibility of staphylococcal planktonic cultures}

The minimum inhibitory concentration (MIC) and minimum bactericidal concentration

(MBC) were evaluated for vancomycin, daptomycin and linezolid according to EUCAST guidelines [8]. For tedizolid, using 96-well plates, $2 \mu \mathrm{L}$ of the relevant $50 \times$ tedizolid was combined with $98 \mu \mathrm{L}$ of an overnight culture adjusted to $5 \times 10^{5} \mathrm{cfu} / \mathrm{mL}$. S. aureus ATCC 
29213 was included as a control strain; all results were within guideline limits. MIC/MBCs were repeated independently at least three times.

\subsection{Time-kill assays}

The time-kill kinetics were determined for two MRSA isolates; one linezolid-sensitive and one linezolid-resistant. Overnight cultures were diluted to a final concentration of $1 \times 10^{6}$ $\mathrm{cfu} / \mathrm{mL}$ in $50 \mathrm{~mL}$ fresh MHB (antibiotic-free control) and MHB supplemented with each antibiotic (tedizolid, linezolid and vancomycin) at a concentration of $0.25 \times, 1 \times$ and $10 \times \mathrm{MIC}$ and then incubated at $37^{\circ} \mathrm{C}$ with aeration at 200rpm for $24 \mathrm{~h}$. Aliquots of $1 \mathrm{~mL}$ were removed at time zero and then every 30 minutes for the first $6 \mathrm{~h}$ and finally $24 \mathrm{~h}$ postinoculation and viable counts obtained. Experiments were performed in triplicate.

\subsection{Antibiotic susceptibility of biofilms}

Twenty robust biofilm forming strains, selected using the crystal violet staining technique (data not shown), were evaluated for antibacterial susceptibility whilst in a biofilm mode of growth; 5 each MRSA, MSSA, S. epidermidis and linezolid-resistant Staphylococcus strains.

Overnight cultures adjusted to $1 \times 10^{6} \mathrm{cfu} / \mathrm{mL}$ were inoculated into 96 -well plates and incubated for $24 \mathrm{~h}$ at $37^{\circ} \mathrm{C}$ on a rocking platform (60 oscillations/min). Then, supernatants were removed, biofilms washed three times with phosphate buffered saline (PBS, Oxoid) and $150 \mu \mathrm{L}$ of antibiotic supplemented MHB added at concentrations of $0.25 \times, 1 \times, 10 \times$ or 100xMIC (except where 100×MIC exceeded $C_{\max }$ ). Antibiotic-free controls were included. After $24 \mathrm{~h}$ antibiotic exposure at $37^{\circ} \mathrm{C}, 0.001 \%(\mathrm{v} / \mathrm{v})$ resazurin (Sigma) in PBS was added to each washed biofilm and incubated at $37^{\circ} \mathrm{C}$ in the dark for $2 \mathrm{~h}$, then fluorescence measured $\left(\mathrm{EM}_{590 \mathrm{~nm}} / \mathrm{EX}_{540 \mathrm{~nm}}\right)$ using a plate reader (FLUOstar Optima, BMG Labtech, Germany), 
providing an indirect measure of the viable cells. The experiment was repeated on two further occasions. Any significant outliers among technical replicates were determined using

150 Grubbs' test ( $p$-value $<0.05$ ) and excluded from further analysis. Using the fluorescence readings, the percentage of cells surviving within an antibiotic-treated biofilm was determined by comparison with the untreated control. Statistical difference between treated and untreated biofilms was determined using Student's t-test and GraphPad Prism 7 Software.

\subsection{Tedizolid susceptibility of biofilms under flow-conditions}

A flow-cell system was used to evaluate susceptibility under conditions replicating the in vivo environment. Three silicone coupons were place in each of two chambers of a FC 275 flow-cell (BioSurface Technologies Corporation, Montana, USA) and MHB introduced into the system via two reservoirs. Using overnight cultures of MSSA31, the coupons were inoculated with $1 \times 10^{6} \mathrm{cfu} / \mathrm{mL}$ and maintained under static conditions for $1 \mathrm{~h}$ at $37^{\circ} \mathrm{C}$ to aid attachment, and then media flow $(1 \mathrm{~mL} /$ minute) continued for 3 days during biofilm formation. Subsequently, one reservoir was replaced with fresh MHB (antibiotic-free control) and the second with MHB supplemented with 10×MIC tedizolid, linezolid or vancomycin and flow resumed for a further $24 \mathrm{~h}$ at $37^{\circ} \mathrm{C}$. Finally, the coupons were removed, rinsed and individually sonicated $3 \times 5$ minutes in PBS using a sonicating waterbath and viable counts determined. Each experiment was performed either in duplicate or triplicate. Percentage cell survival was calculated (section 2.4) and statistical difference between treated and untreated biofilms determined using Student's t-test. 


\subsection{Antibiotic susceptibility of planktonic cultures}

173 The MICs and MBCs were determined against the 66 staphylococci (Supplemental Data Table 1). There was no evidence of resistance to any of the antibiotics tested, except for the 175 Spanish strains that were resistant to linezolid, and all the MIC ranges and $\mathrm{MIC}_{50}$ values for linezolid, vancomycin and daptomycin were as expected being within one-dilution of the EUCAST published data [8] (Supplemental Data Table 1). All the linezolid-sensitive strains were highly susceptible to tedizolid with MICs within the narrow range of $0.125-0.5 \mathrm{mg} / \mathrm{L}$; a median MIC value 8-fold below that of linezolid. The tedizolid sensitivity of linezolidresistant strains varied with the resistance mechanism; those $c f r+$ had a tedizolid MIC of 0.25-0.5 $\mathrm{mg} / \mathrm{L}$ (versus $8 \mathrm{mg} / \mathrm{L}$ linezolid), those that possessed the G2576T mutation had tedizolid MIC values of 2-4 mg/L (versus $16-64 \mathrm{mg} / \mathrm{L}$ linezolid), whilst the strain exhibiting both linezolid-resistance mechanisms had MIC values of $4 \mathrm{mg} / \mathrm{L}$ tedizolid and $512 \mathrm{mg} / \mathrm{L}$ linezolid.

Vancomycin and daptomycin were shown to be bactericidal, with only $16 \%$ and $<1 \%$ of isolates presenting with a MBC:MIC ratio $\geq 8$. By contrast linezolid and tedizolid were bacteriostatic (Supplemental Data Table 1).

\subsection{Time-kill kinetics}

Time-kill kinetics were determined for tedizolid, linezolid and vancomycin for two MRSA strains; one sensitive (MRSA23) and one resistant ( $\left.\mathrm{frr}^{+} \mathrm{JMO2}\right)$ to linezolid (Fig.1). Sub-MIC antibiotic exerted minimal effect on the growth of the organisms with viable bacterial cell 
bacteriostatic against both isolates with activity against the linezolid-resistant strain comparable to that exerted against the sensitive strain (Fig.1). Despite initially impeding growth, $1 \times \mathrm{MIC}$ linezolid failed to inhibit growth of $c f r^{+} \mathrm{JMO} 02$ with a 2-log increase in bacterial cell number compared to the initial inoculum after $24 \mathrm{~h}$ exposure to the agent (10xMIC exceeded the therapeutically achievable concentration and was not tested). Conversely, a >3-log reduction in viable cell number in comparison to the initial inoculum was attained after $24 \mathrm{~h}$ exposure to $1 \times$ and $10 \times$ MIC vancomycin confirming the bactericidal nature of the agent.

\subsection{Antibiotic susceptibility of biofilms}

A dose-dependent response was noted for biofilms challenged with each antibacterial. At a concentration of $1 \times \mathrm{MIC}$, no agent was able to reduce the proportion of viable cells within the biofilm to $60 \%$ or fewer of untreated control biofilm; vancomycin in particular had little if any impact (Fig.2a). The mean level of activity exerted against S. epidermidis isolates (77$107 \%$ mean survival) by each antibacterial was inferior to that exhibited against the $S$. aureus isolates (64-103\% mean survival), an effect that was of particular note with linezolid (103\% vs 64\% mean survival, S. epidermidis and S. aureus respectively).

When challenged with $10 \times \mathrm{MIC}$ antibacterial there was a marked reduction in the proportion of cells within the biofilm remaining viable (28-77\% mean cells remaining viable) (Fig.2b).

Against S. aureus all of the agents tested reduced the biofilm to below a 50\% mean of the untreated control (28-45\% mean cell survival) compared to S. epidermidis where only vancomycin and tedizolid attained a comparable reduction ( 29 and $35 \%$ mean cell survival, respectively); linezolid and daptomycin achieved only a $33 \%$ and $47 \%$ mean decrease in 
219 viable cells, respectively, with the majority of cells remaining viable after treatment. In

220 addition to the greater level of tolerance exhibited by S. epidermidis isolates, there was also

221 greater variation in susceptibility between the strains. At least one S. epidermidis isolate

222 was unaffected by $10 \times$ MIC of any agent tested ( $97-103 \%$ cell viability), the exception being

223 tedizolid which retained a good level of activity against all the S. epidermidis strains tested,

224 including the linezolid-resistant strains.

225

226 Under flow-conditions tedizolid was superior to vancomycin and comparable to linezolid in

227 the ability to reduce the proportion of viable biofilm-associated cells remaining on the

228 silicone coupons (Fig.3). Exposure to 10xMIC tedizolid or linezolid led to a statistically

229 significant reduction in the proportion of viable cells remaining within the biofilm $(8 \%, p$

$230<0.05$ or $12 \%, p<0.005$ cell survival compared to the untreated control, respectively) while

231 vancomycin did not achieve a statistically significant reduction in viable cells $(63 \%, p=0.08)$. 


\section{Discussion and Conclusion}

234 Ranging from mild to life-threatening, SSTI are among the most commonly occurring infections and evidence suggests that these are increasing. From 1993-2005, the number of emergency department visits in the USA by patients with these presentations increased from 1.2 million visits to 3.4 million [9]. Whilst SSTIs are diverse in aetiology, S. aureus are consistently predominating world-wide with multi-drug resistant strains increasingly being reported [10]. In the USA, one study reported that $81 \%$ of culture-positive SSTIs were caused by S. aureus, with almost half (46\%) of those strains recovered being resistant to methicillin [11]. The high prevalence of the USA300 MRSA strain may account in part for these figures. In Europe however, where USA300 remains typically rare, a similar profile is economic burden to healthcare providers.

Achieving an effective treatment combining surgical debridement or drainage with empirical antibiotic therapy is not without its challenges. From the microbiological prospective, the agent is often unknown, multidrug-resistance is prevalent and a biofilm mode of growth complicates therapy. Though more prevalent in chronic wounds with $60 \%$ of samples being positive, biofilms have also been detected in $6 \%$ of acute wounds [13]. In this study, the increased activity of tedizolid compared to linezolid and other anti-staphylococcal agents was achieved typically using lower concentrations against both planktonic and biofilmassociated cells, including $c f r+$ multidrug-resistant strains. Against S. aureus biofilms, tedizolid was superior or comparable with comparator agents in activity, and typically superior against those formed by S. epidermidis strains. Under flow-conditions mimicking 
the in vivo environment of a SSTI, for example infection related to an indwelling-device, the bacteriostatic oxazolidinones both out-performed vancomycin.

Vancomycin has been a mainstay of treatment for staphylococcal SSTI. However, the decreased susceptibility observed in vancomycin intermediate susceptibility S. aureus (VISA) and strains displaying heteroresistance, the need for intravenous slow-infusion and to monitor serum levels, and the potential for toxicity have led to moves towards other antibacterial agents. The alternatives currently available include linezolid, telavancin and daptomycin. Linezolid has been shown to be more effective at treating SSTIs than vancomycin with fewer complications being reported and patient discharge occurring sooner [14]. Whilst linezolid retains a good level of activity against staphylococci [15], the emergence of linezolid resistance in staphylococci is a concern [16]. Tedizolid has however, been demonstrated by this study to retain activity against $c f r+$ staphylococci. It has previously been reported that the sterically compact nature of the hydroxymethyl group of tedizolid greatly improves activity against strains possessing the $c f r$ gene [6]. In addition, tedizolid was reported by Russo et al. (2016) to be statistically non-inferior to linezolid in patients with SSTI for an early clinical response evaluated $48-72 \mathrm{~h}$ after beginning therapy [17]. Other factors favouring the use of tedizolid over contemporary agents in the treatment of SSTI include the long half-life (double linezolid) allowing once a day dosing, short course these studies are undertaken in vitro and as such cannot infer in vivo activity, however, the data generated suggest that tedizolid offers an additional drug choice for the treatment of SSTIS. 
In conclusion, in this in vitro study the anti-staphylococcal activity of tedizolid has been shown to be at least comparable and often superior to comparator agents that are routinely prescribed in the treatment of SSTIs. Taken with the drive to de-escalate SSTI treatment sooner, switching early to a short-course oral agent allowing early discharge, tedizolid offers a realistic lower dose alternative agent in the treatment of staphylococcal SSTI, including those where biofilms are present.

287

\section{Acknowledgements}

The authors would like to thank MSD/Merck for funding for this research and the supply of tedizolid. We also thank Pfizer for the kind gift of the linezolid. In addition we would like to thank J. Mingorance (Hospital Universitario La Paz, Madrid), Dr Elizabeth Dickson, Scottish MRSA Reference Laboratory (SMRSARL), Glasgow Royal Infirmary, Glasgow, for the supply of strains.

294

Some of the data presented in this manuscript has previously been reported at the 27 th

European Congress of Clinical Microbiology and Infectious Diseases (ECCMID), Vienna 22-25

297 April 2017 and the Microbiology Society Annual Conference 2017, Glasgow 3 - 6 April 2017.

\section{Declarations}

300 Funding: This work was supported MSD/Merck (MISP\#53795). The funders had no role in 301 study design, data collection or analysis. 
303 Ethical Approval: Work involving the transconjugate strain Staphylococcus aureus ATCC

$30429213 \mathrm{cfr}^{+}$was performed under The Genetically Modified Organisms (Contained Use)

305 Regulation 2014 of the Health and Safety Executive. 
307 [1] Bassetti M, Baguneid M, Bouza E, Dryden M, Nathwani D, Wilcox M. European 308 perspective and update on the management of complicated skin and soft tissue infections due to methicillin-resistant Staphylococcus aureus after more than 10 years of experience with linezolid. Clinical microbiology and infection : the official publication of the European Society of Clinical Microbiology and Infectious Diseases. 2014;20 Suppl 4:3-18.

[2] Zervos MJ, Freeman K, Vo L, Haque N, Pokharna H, Raut M, et al. Epidemiology and outcomes of complicated skin and soft tissue infections in hospitalized patients. Journal of clinical microbiology. 2012;50:238-45.

[3] McClain SL, Bohan JG, Stevens DL. Advances in the medical management of skin and soft tissue infections. Bmj. 2016;355:i6004.

[4] McCool R, Gould IM, Eales J, Barata T, Arber M, Fleetwood K, et al. Systematic review and network meta-analysis of tedizolid for the treatment of acute bacterial skin and skin structure infections caused by MRSA. BMC infectious diseases. 2017;17:39.

[5] Merck. Sivextro (tedizolid phosphate) for injection, for intravenous use; Sivextro (tedizolid phosphate) tablet, for oral use. Merck \& Co, Whitehouse Station, NJ2015. [6] Locke JB, Finn J, Hilgers M, Morales G, Rahawi S, G CK, et al. Structure-activity relationships of diverse oxazolidinones for linezolid-resistant Staphylococcus aureus strains possessing the cfr methyltransferase gene or ribosomal mutations. Antimicrobial agents and chemotherapy. 2010;54:5337-43.

[7] Rybak JM, Roberts K. Tedizolid Phosphate: a Next-Generation Oxazolidinone. Infectious diseases and therapy. 2015.

[8] EUCAST. European Committee on Antibacterial Susceptibility Testing. 2017.

[9] Pallin DJ, Egan DJ, Pelletier AJ, Espinola JA, Hooper DC, Camargo CA, Jr. Increased US emergency department visits for skin and soft tissue infections, and changes in antibiotic choices, during the emergence of community-associated methicillin-resistant Staphylococcus aureus. Annals of emergency medicine. 2008;51:291-8.

[10] Moet GJ, Jones RN, Biedenbach DJ, Stilwell MG, Fritsche TR. Contemporary causes of skin and soft tissue infections in North America, Latin America, and Europe: report from the SENTRY Antimicrobial Surveillance Program (1998-2004). Diagnostic microbiology and infectious disease. 2007;57:7-13.

[11] Ray GT, Suaya JA, Baxter R. Incidence, microbiology, and patient characteristics of skin and soft-tissue infections in a U.S. population: a retrospective population-based study. BMC infectious diseases. 2013;13:252.

[12] Morrissey I, Leakey A, Northwood JB. In vitro activity of ceftaroline and comparator antimicrobials against European and Middle East isolates from complicated skin and skinstructure infections collected in 2008-2009. International journal of antimicrobial agents. 2012;40:227-34.

[13] James GA, Swogger E, Wolcott R, Pulcini E, Secor P, Sestrich J, et al. Biofilms in chronic wounds. Wound repair and regeneration : official publication of the Wound Healing Society [and] the European Tissue Repair Society. 2008;16:37-44.

[14] Yue JD, BR; Yang, M; Chen, X; Wu, T; Liu, G. Linezolid versus vancomycin for skin and soft tissue infections. Cochrane Library. 2016.

[15] Pfaller MA, Mendes RE, Streit JM, Hogan PA, Flamm RK. Five-Year Summary of In Vitro Activity and Resistance Mechanisms of Linezolid against Clinically Important GramPositive Cocci in the United States from the LEADER Surveillance Program (2011 to 2015). Antimicrobial agents and chemotherapy. 2017;61.

[16] Quiles-Melero I, Gomez-Gil R, Romero-Gomez MP, Sanchez-Diaz AM, de Pablos M, Garcia-Rodriguez J, et al. Mechanisms of linezolid resistance among Staphylococci in a tertiary hospital. Journal of clinical microbiology. 2013;51:998-1001. 
[17] Russo A, Concia E, Cristini F, De Rosa FG, Esposito S, Menichetti F, et al. Current and future trends in antibiotic therapy of acute bacterial skin and skin-structure infections.

358 Clinical microbiology and infection : the official publication of the European Society of 359 Clinical Microbiology and Infectious Diseases. 2016;22 Suppl 2:S27-36.

360

361 
Figure Legends

Figure 1. Time-kill kinetics of linezolid sensitive MRSA23 (a,c,e) and linezolid resistant MRSA $(\mathrm{cfr}+)$ JM02 (b,d,f) challenged with $(\mathrm{a}, \mathrm{b})$ tedizolid, $(\mathrm{c}, \mathrm{d})$ linezolid and $(e, f)$ vancomycin at concentrations of $0.25 \times \mathrm{MIC}(\bullet), 1 \times \mathrm{MIC}(\Delta), 10 \times \mathrm{MIC}(\boldsymbol{\nabla})$, compared to untreated control cultures $(\bullet)$. Error bars represent SEM between replicate samples $(n=3)$. Broken line $(\ldots$. indicates a 3-log reduction in viable cell number in comparison to the initial inoculum. cfu, colony forming units.

Figure 2. Susceptibility of biofilm-associated staphylococcal cells exposed to (a) $1 \times$ MIC or (b) 10×MIC antibiotic compared to untreated control cultures. Antibiotics included VAN, vancomycin; DAP, daptomycin; LZD, linezolid; TZD, tedizolid. Cell survival was assessed using the metabolic dye resazurin. Each experiment consisted of four replicate biofilms and was repeated a further two times. Error bars represent SEM.

Figure 3. Susceptibility of MSSA31 biofilm-associated cells cultivated on silicone rubber coupons and exposed to 10xMIC antibiotic under flow conditions within a BST flow-cell system. Antibiotics included VAN, vancomycin; LZD, linezolid; and TZD, tedizolid. Paired ttest; $* *$-value $<0.05 ; * * * *$-value $<0.005 ;$ no asterisk $p>0.05$. Each experiment consisted of three replicate silicone rubber coupons and a minimum of two independent repeats. 
Tedizolid

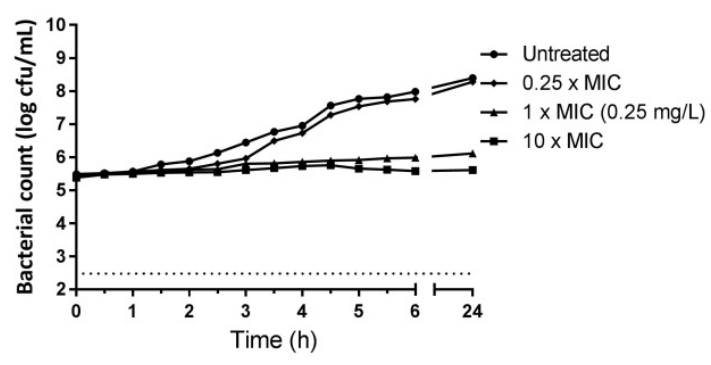

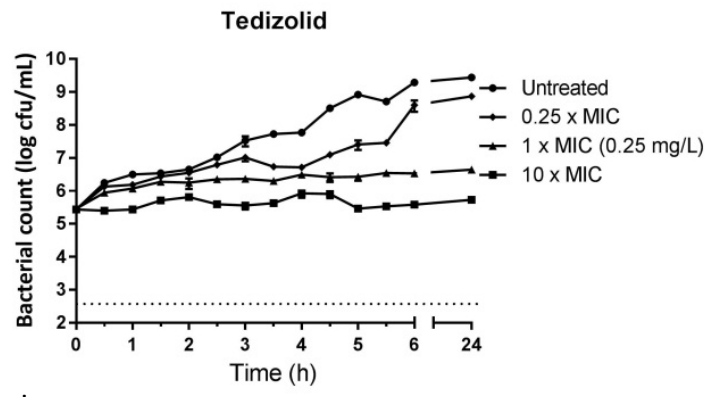

b
Linezolid

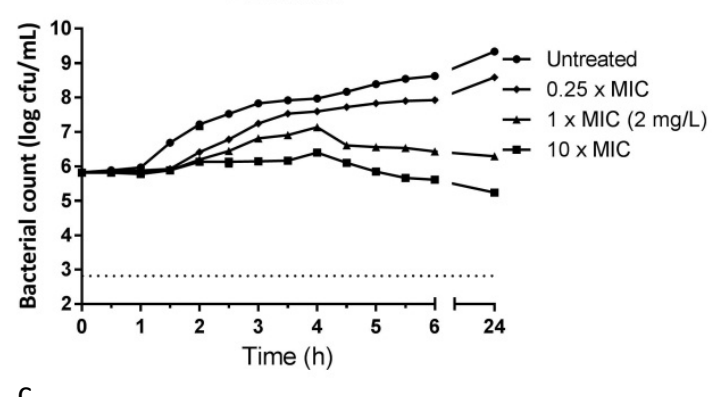

C

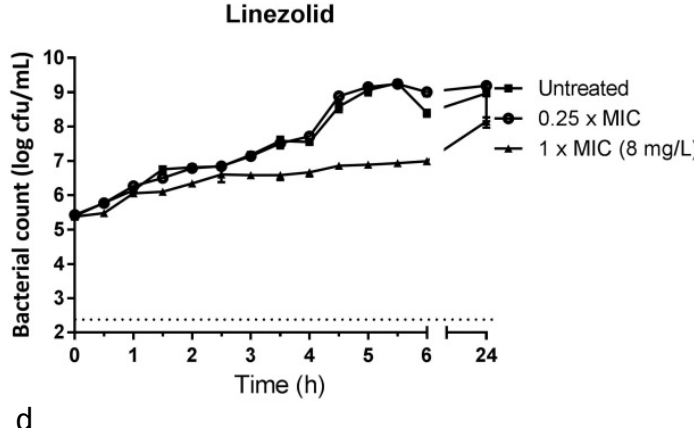

d
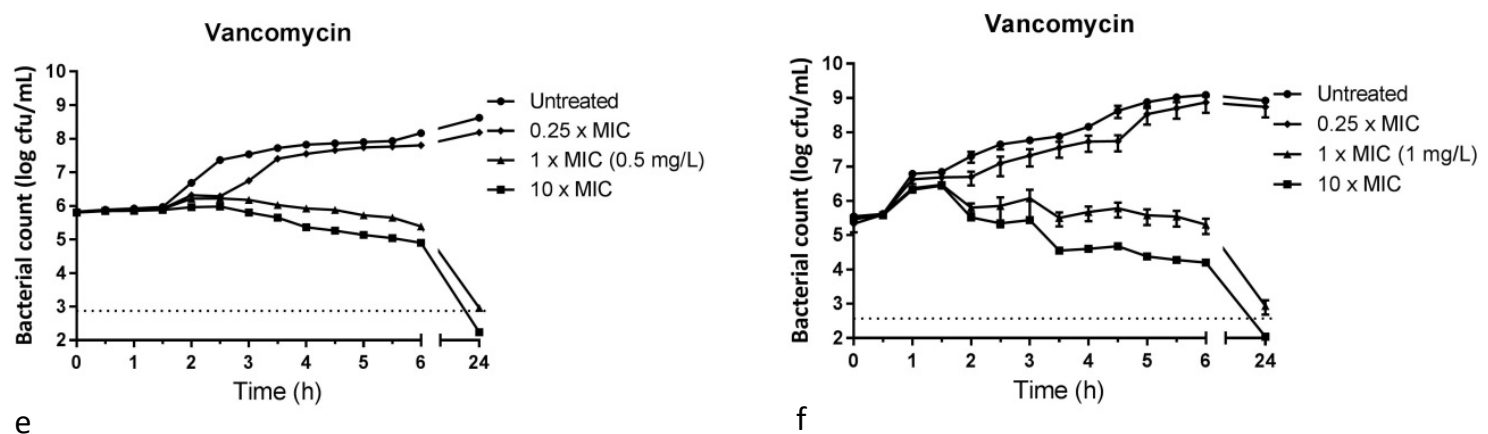

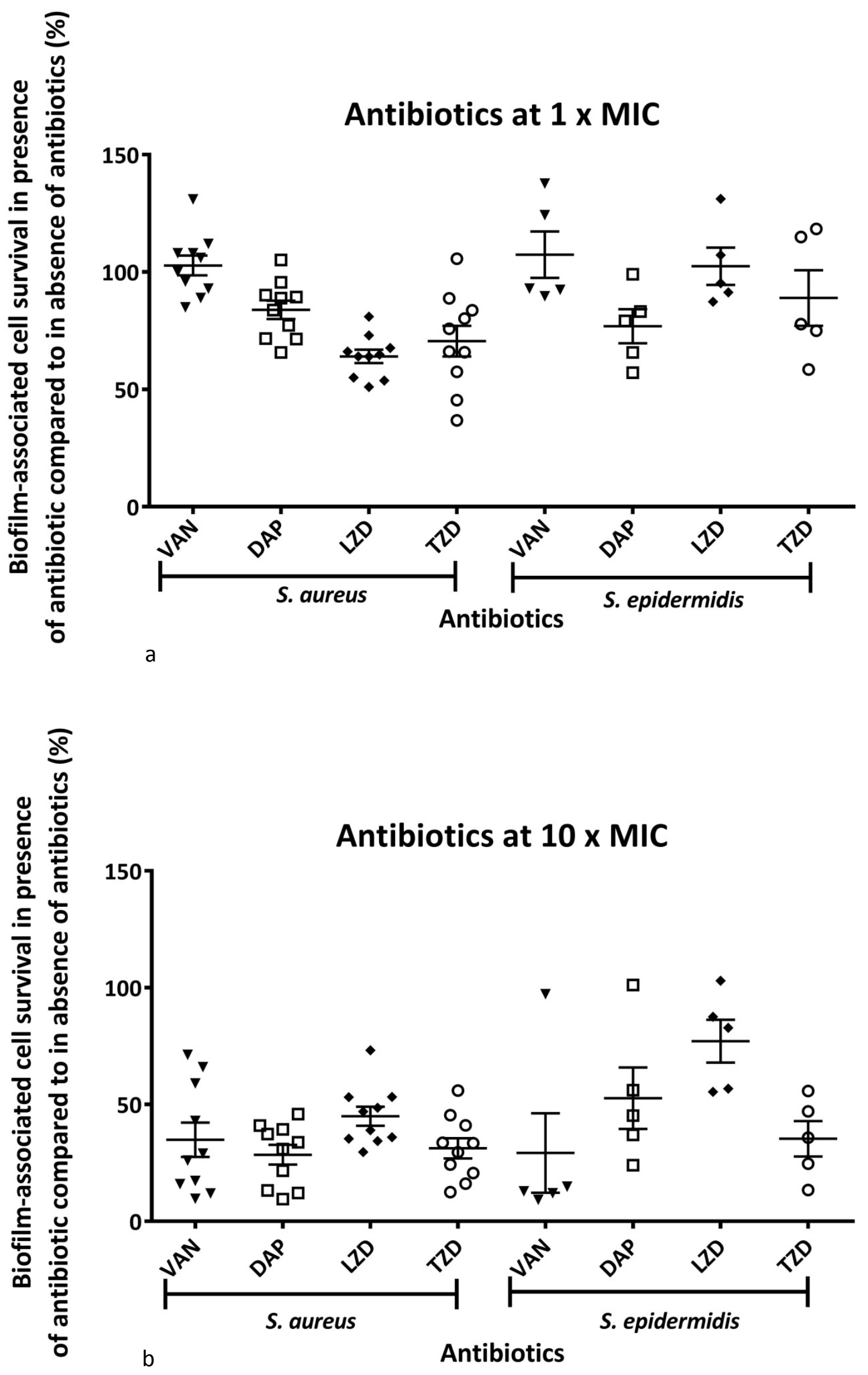


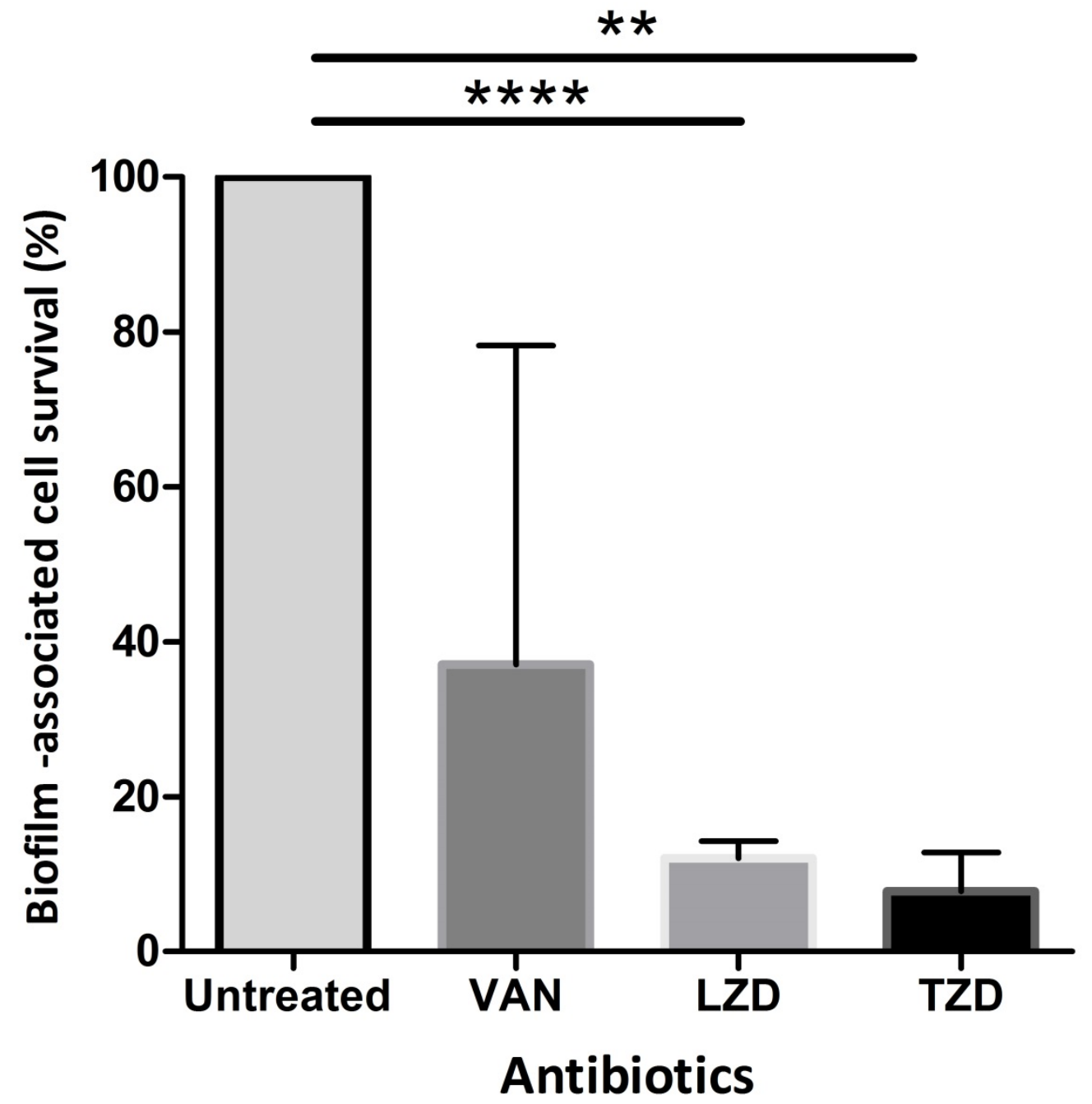


Supplementary Table 1. Origin of the strains used in this study.

\begin{tabular}{|c|c|c|c|c|c|}
\hline $\begin{array}{l}\text { Staphylococcus } \\
\text { species }\end{array}$ & Strain & Sample type & Origin $^{a}$ & Isolated & Comments \\
\hline \multirow{3}{*}{$\begin{array}{c}\text { Methicillin resistant } \\
\text { S. aureus } \\
\text { (MRSA) } \\
\mathrm{n}=27\end{array}$} & MRSA1 - 25 & Blood & Scottish hospitals & $2014-2015$ & spa typed ${ }^{\mathrm{b}}$ \\
\hline & JM01 & $\mathrm{nk}$ & Madrid, Spain & $\mathrm{nk}$ & Linezolid resistant $\left(c f r^{+}\right)$ \\
\hline & JM02 & nk & Madrid, Spain & nk & Linezolid resistant $\left(c f r^{+}\right)$ \\
\hline \multirow{4}{*}{$\begin{array}{l}\text { Methicillin sensitive } \\
\qquad \begin{array}{c}\text { S. aureus } \\
\text { (MSSA) } \\
\mathrm{n}=27\end{array}\end{array}$} & MSSA25 - 50 & Blood & Scottish hospitals & 2014-2015 & spa typed ${ }^{b}$ \\
\hline & JM03 & nk & Madrid, Spain & $\mathrm{nk}$ & Linezolid resistant (G2576T mutation) \\
\hline & JM04 & nk & Madrid, Spain & - & $\begin{array}{l}\text { Linezolid resistant GMO; } c f^{+} \text {transconjugant of } \\
\text { strain ATCC } 29213\end{array}$ \\
\hline & ATCC 29213 & - & Reference strain & - & Antibiotic sensitivity control strain \\
\hline \multirow{3}{*}{$\begin{array}{l}\text { S. epidermidis } \\
\quad \mathrm{n}=12\end{array}$} & JM05 & nk & Madrid, Spain & nk & Linezolid resistant (G2576T mutation) \\
\hline & JM06 & $\mathrm{nk}$ & Madrid, Spain & nk & Linezolid resistant ( $c f r^{+}$and G2576T mutation) \\
\hline & $\begin{array}{c}10,70,93,96,103,105 \\
117,122,157,178\end{array}$ & $\begin{array}{l}\text { Various } \\
\text { clinical } \\
\text { isolates }\end{array}$ & Scottish hospitals & 2011-13 & 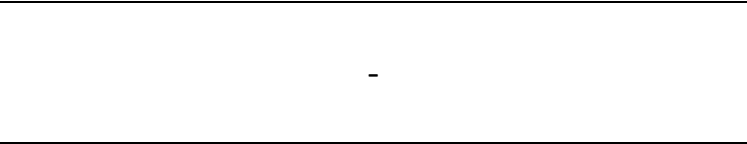 \\
\hline
\end{tabular}

; Hospital Universitario La Paz, Madrid or Scottish MRSA Reference Laboratory (SMRSARL), Glasgow Royal Infirmary, Glasgow.

${ }^{\mathrm{b}}$; Isolates represented 32 different spa types (1-9 representatives per spa type with t032 being predominant) and 12 clonal complexes (with CC22, $\mathrm{n}=20$; CC5, $n=6$; and CC30, $n=5$ being the principal types).

nk; not known. 
Supplemental Table 2. Antibiotic susceptibility of staphylococci grown in planktonic culture.

\begin{tabular}{|c|c|c|c|c|c|c|c|}
\hline \multirow[t]{2}{*}{ Antibacterial } & \multirow[t]{2}{*}{ Organism } & \multicolumn{6}{|c|}{ Antibiotic susceptibility (mg/l) } \\
\hline & & MIC range & $\mathrm{MIC}_{50}$ & $\mathrm{MIC}_{90}$ & $\mathrm{MBC}_{50}$ & $\mathrm{MBC}_{90}$ & $\mathrm{MBC}_{50} / \mathrm{MIC}_{50}$ \\
\hline \multirow[t]{3}{*}{ Tedizolid } & MSSA & $0.125-2$ & 0.25 & 0.5 & 4 & 4 & 16 \\
\hline & MRSA & $0.125-0.5$ & 0.25 & 0.5 & 2 & 4 & 8 \\
\hline & S. epidermidis & $0.25-4$ & 0.25 & 4 & 4 & $>4$ & 16 \\
\hline \multirow[t]{3}{*}{ Linezolid } & MSSA & $2-16$ & 2 & 4 & 16 & $>16$ & 8 \\
\hline & MRSA & $2-8$ & 2 & 4 & 16 & $>16$ & 8 \\
\hline & S. epidermidis & $1-512$ & 1 & 64 & 32 & 64 & 32 \\
\hline \multirow[t]{3}{*}{ Vancomycin } & MSSA & $0.25-1$ & 0.5 & 1 & 2 & 4 & 2 \\
\hline & MRSA & $0.25-1$ & 0.5 & 1 & 2 & $>8$ & 2 \\
\hline & S. epidermidis & $1-2$ & 1 & 2 & 2 & 4 & 2 \\
\hline \multirow[t]{3}{*}{ Daptomycin } & MSSA & $0.25-1$ & 0.5 & 1 & 1 & 2 & 2 \\
\hline & MRSA & $0.5-1$ & 0.5 & 1 & 1 & 2 & 2 \\
\hline & S. epidermidis & $0.5-2$ & 0.5 & 1 & 0.5 & 2 & 1 \\
\hline
\end{tabular}

MSSA, $n=27$ including 2 linezolid resistant strains; MRSA, $n=27$ including 2 linezolid resistant strains; S. epidermidis, $n=12$ including 2 linezolid resistant strains. 